\section{ASSOCIAÇÃO DA ATIVIDADE FÍSICA DE ADOLESCENTES, FAMILIARES E PARES: UMA REVISÃO SISTEMÁTICA}

\author{
Association of physical activity of adolescents, families and \\ peers: a systematic review
}
Asociación de la actividad física de adolescentes, los familiares y sus pares: una revisión sistemática

Artigo de Revisão

\section{RESUMO}

Objetivo: Analisar a associação entre a atividade física (AF) de pais, irmãos e pares à $\mathrm{AF}$ de adolescentes brasileiros por meio de uma revisão sistemática da literatura. Métodos: Realizou-se revisão sistemática em duas etapas, em junho de 2013 e fevereiro de 2014, nas bases de dados PubMed/MEDLINE, Web of Science, EBSCO e LILACS, utilizando-se quatro grupos de descritores, sendo estes "influência de pais e irmãos", "atividade física", "tipo de amostra" e "nacionalidade", nos idiomas inglês e português, que atendesse aos seguintes critérios: (i) indivíduos saudáveis; (ii) adolescentes brasileiros; (iii) estudos abordando a AF; e (iv) estudos publicados entre janeiro de 2008 e fevereiro de 2014. Resultados: A busca nas bases de dados resultou em 2.094 títulos de artigos potencialmente relevantes, mas apenas 7 atenderam a todos os critérios de inclusão. Há uma associação positiva entre a AF de pais e filhos, porém, ao estratificar por sexo, esta é encontrada apenas no feminino, tanto para a inatividade quanto para estar fisicamente ativo. $\mathrm{O}$ apoio social possui grande influência na AF de crianças e adolescentes, no entanto, tal associação não foi encontrada em estudos longitudinais. Conclusão: Existe uma associação positiva entre a atividade física do pai e da mãe com suas filhas, tanto para a inatividade quanto para serem ativos, mas não foram encontradas associações entre os pais e os filhos, fato também observado para com irmãos inativos. O apoio social mostrou-se eficaz para aumentar o nível de atividade física de adolescentes.

Descritores: Atividade Motora; Relações Familiares; Amigos.

\section{ABSTRACT}

Objective: To analyze the association between physical activity (PA) of parents, siblings and peers with the PA of Brazilian adolescents through a systematic review. Methods: We conducted a systematic review in two stages, in June 2013 and February 2014 in the PubMed/ MEDLINE, Web of Science, EBSCO and LILACS databases using four sets of descriptors, those being the influence of parents and siblings, physical activity, type of sample, and nationality, in English and Portuguese, which should meet the following criteria: (i) healthy subjects; (ii) Brazilian adolescents; (iii) studies addressing PA; and (iv) studies published between January 2008 and February 2014. Results: The search in databases resulted in 2,094 titles of potentially relevant articles, but only seven met all the inclusion criteria. There is a positive association between parents' and children's $P A$; however, when stratified by sex, this association is found only in women, both for inactivity and for being physically active. Social support has great influence on the PA of children and adolescents; however, such association has not been found in longitudinal studies. Conclusion: There is a positive association between the PA of father and mother with that of their daughters, both for inactivity and for being active, but no association was found between parents and sons, a fact that has also been observed as to inactive siblings. Social support proved to be effective to increase the level of physical activity in adolescents.
Recebido em: 11/05/2015 Revisado em: 20/05/2015 Aceito em: 29/05/2015 


\section{RESUMEN}

Objetivo: Analizar la asociación entre la actividad física $(A F)$ de los padres, los hermanos y sus pares con la AF de adolescentes brasileños a través de una revisión sistemática de la literatura. Métodos: Se realizó una revisión sistemática en dos etapas distintas (junio de 2013 y otra en febrero de 2014) en las bases de datos PubMed/MEDLINE, Web of Science, EBSCO y LILACS utilizándose cuatro grupos de descriptores "influencia de los padres y hermanos", "actividad fisica", "tipo de muestra" y "nacionalidad" en los idiomas inglés y portugués que atendiera a los siguientes criterios: (i) individuos saludables; (ii) adolescentes brasileños; (iii) estudios sobre la AF; y (iv) estudios publicados entre enero de 2008 y febrero de 2014. Resultados: La búsqueda en las bases de datos resultó en 2.094 títulos de artículos relevantes en potencial, pero solamente 7 cumplieron todos los criterios de inclusión. Hay una asociación positiva entre la AF de padres e hijos, pero al estratificarlos por el sexo, la asociación se da solamente para el femenino tanto para la inactividad como para estar fisicamente activo. El apoyo social tiene gran influencia en la AF de niños y adolescentes, sin embargo, esta asociación no ha sido encontrada en estudios longitudinales. Conclusión: Existe una asociación positiva entre la actividad fisica del padre y de la madre con sus hijas para la inactividad y para el hecho de ser fisicamente activo pero no fueron encontradas asociaciones entre los padres e hijos, hecho observado también con los hermanos inactivos. El apoyo social se mostró eficaz para el aumento del nivel de la actividad fisica de los adolescentes.

Descriptores: Actividad Motora; Relaciones Familiares; Amigos.

\section{INTRODUÇÃO}

Os benefícios de uma prática regular de atividades físicas (AF) estão bem esclarecidos pela literatura científica, a qual aponta inúmeros benefícios ligados à adoção de um estilo de vida ativo, dentre eles, a melhora da força e flexibilidade, prevenção de doenças metabólicas, bem-estar psicológico e controle do peso corporal ${ }^{(1-6)}$. Todavia, para que esses benefícios possam ser alcançados, a AF deve estar presente em pelo menos 60 minutos do $\mathrm{dia}^{(7)}$, conforme sugerido pela literatura $^{(8-10)}$.

Atualmente, estima-se que $31 \%$ da população mundial não esteja enquadrada dentro das recomendações mínimas para $\mathrm{AF}$, e que $80,3 \%$ dos jovens entre 13 e 15 anos também não cumpram as recomendações pertinentes à sua faixa etária ${ }^{(11)}$. Além do alarmante problema de crianças e adolescentes apresentarem uma tendência a diminuir seus níveis de AF no decorrer dos anos ${ }^{(12-14)}$, apresentando-se como um considerável fator de risco para desordens metabólicas, as quais tendem a permanecer na fase adulta ${ }^{(15,16)}$. Sobre esse aspecto, o problema dos baixos níveis de atividade física (NAF) fica ainda mais preocupante, considerando que, mesmo dentro dos padrões recomendados para a AF, as evidências estão apontando para o fato de que o tempo de atividades sedentárias, quando em excesso, pode aumentar o risco de mortalidade e doenças cardiovasculares ${ }^{(5,17)}$.

A opção por um estilo de vida sedentário na infância e adolescência, em parte, é influenciada pelos pais ${ }^{(1,18)}$, porém, o contrário também está descrito pela literatura, ou seja, uma adoção de hábitos saudáveis pode ser influenciada pelos pais ${ }^{(1,19)}$. Nas relações familiares, os irmãos também apresentam correlação nos seus NAFs, tanto para os ativos quanto para o sedentarismo, indicando que sujeitos de uma mesma geração, genética ou intimamente relacionados, tendem a ser similares nos seus hábitos de $\mathrm{AF}^{(20)}$. Além da associação em AF em conjunto dos amigos, que se mostra consistente $^{(21)}$.

Compreender as causas de um comportamento fisicamente ativo é essencial para o desenvolvimento e melhoria das intervenções de saúde pública. Dentre essas causas, as relações sociais são apontadas como fatores que podem afetar a $\mathrm{AF}^{(22)}$.

Assim, o objetivo deste estudo foi analisar a associação entre a atividade física (AF) de pais, irmão e pares, e a AF de adolescentes brasileiros, por meio de uma revisão sistemática da literatura.

\section{MÉTODOS}

Realizou-se uma revisão sistemática nas bases de dados PubMed/MEDLINE, Web of Science, EBSCO e LILACS. A estratégia de busca dos estudos baseou-se em quatro grupos de descritores, sendo estes: influência de pais e irmãos, atividade física, tipo de amostra e nacionalidade, realizada com descritores nos idiomas português e inglês, previamente consultados nos websites Descritores em Ciências da Saúde (DeCS) e Medical Subject Headings (MeSH), respectivamente.

Os descritores utilizados para a busca de estudos potencialmente relevantes consistiram em: para a influência de pais e irmãos (influência OR pais OR parental OR relações familiares OR pares OR irmão), (influence OR parents OR parental OR family relations OR peer OR sibling); para sua combinação com atividade física (atividade motora OR atividade física OR exercício), (motor activity OR physical activity OR exercise); para a combinação com o tipo de amostra (criança OR juventude OR jovem OR adolesc*), (child OR youth OR teen* OR adolesc*) e, por fim, a nacionalidade (Brasil*, Brazil*). Utilizou-se o operador booleano " $A N D$ ” para a combinação entre os grupos de descritores.

A presente revisão sistemática se deu por uma busca em duas etapas, a primeira realizada em junho de 2013 
e a segunda, em fevereiro de 2014, para uma possível atualização das publicações.

Os critérios de inclusão adotados nesta revisão constituíram em: (i) indivíduos saudáveis; (ii) amostras incluindo adolescentes brasileiros de 10 a 19 anos sendo comparados aos seus respectivos familiares; (iii) estudos abordando a AF, independentemente se como variável de exposição ou desfecho; e (iv) estudos publicados no intervalo de tempo entre janeiro de 2008 e fevereiro de 2014, visando a utilização de literatura recente.

Estudos realizados com sujeitos não saudáveis, com algum tipo de deficiência, seja esta momentânea ou permanente; amostras que não fossem brasileiras; estudos que não abordaram a $\mathrm{AF}$, tanto como variável independente quanto dependente; estudos publicados em períodos diferentes dos pré-estabelecidos e publicados em idiomas diferentes do português, inglês ou espanhol foram excluídos.

\section{RESULTADOS}

A pesquisa bibliográfica identificou 2.094 títulos de artigos potencialmente relevantes para a presente revisão, sendo 1.995 na primeira busca e 99 na segunda. Após a leitura de todos os títulos, 109 estudos foram selecionados seguindo os critérios de inclusão e exclusão para terem seus resumos verificados. Após essa etapa, 26 estudos acabaram selecionados para serem lidos na íntegra. Ao final desse processo, 6 artigos atenderam a todos os critérios de inclusão, além de 1 artigo selecionado a partir da lista de referências dos estudos selecionados, totalizando 7 artigos utilizados (Figura 1).

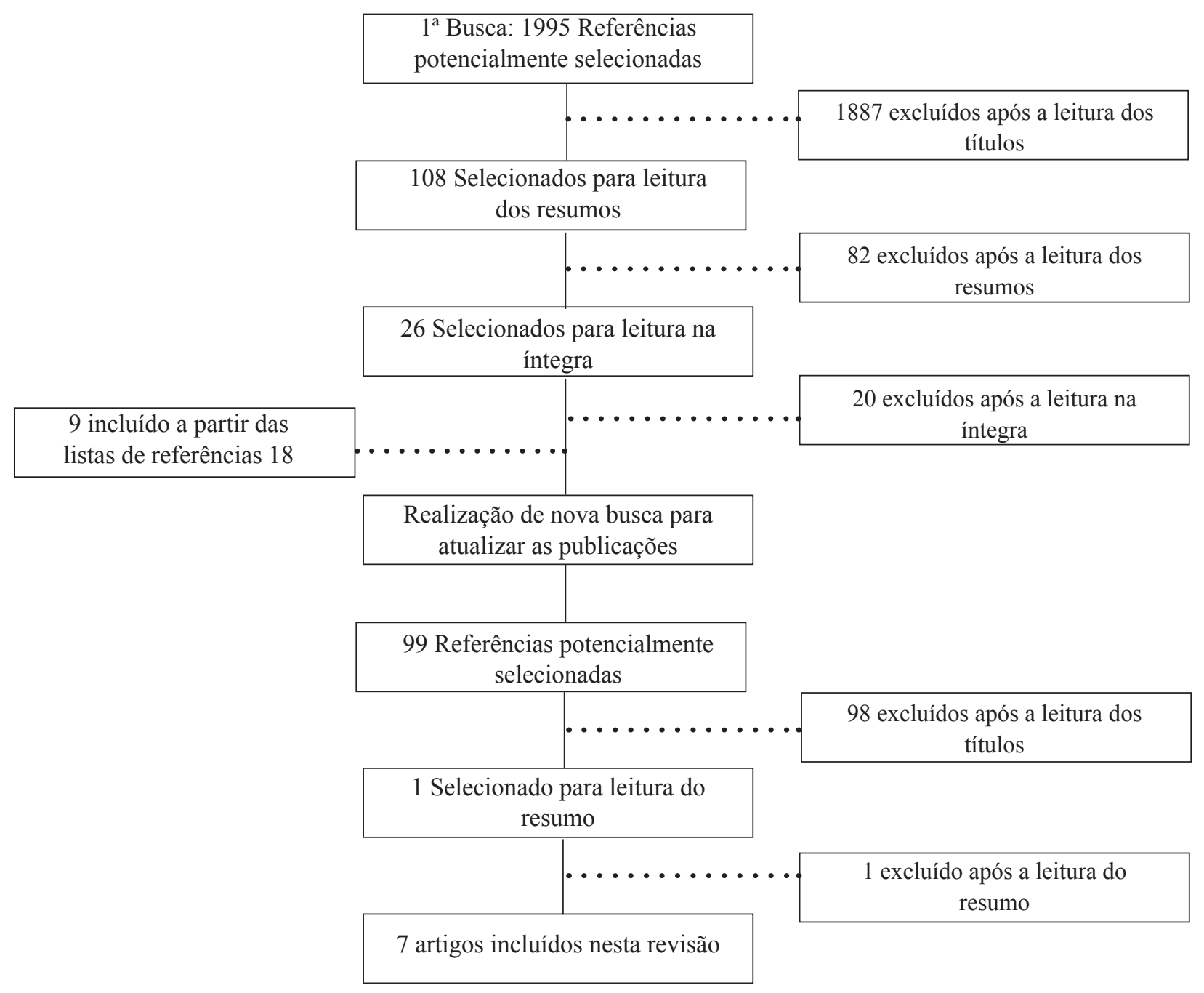

Figura 1 - Fluxograma de seleção dos artigos. Curitiba-PR, 2013-2014. 
O Quadro 1 apresenta as informações gerais sobre as metodologias empregadas, e o Quadro 2 apresenta os principais resultados encontrados pelos 7 artigos incluídos na revisão sistemática. Dos artigos selecionados, seis apresentam design transversal ${ }^{(1,18,23-26)}$ e um, longitudinal prospectivo $^{(14)}$.

As faixas etárias eram próximas entre os estudos, no entanto, a amostra mais jovem era de $7 \operatorname{anos}^{(24)}$ e a mais velha, de 19 anos $^{(23)}$. Porém, a coincidência fica para os estados onde ocorreram as pesquisas. O Rio Grande do Sul esteve presente em quatro estudos, representado pelos municípios de Barão do Triunfo $^{(1)}$ e Pelotas $^{(14,23,26)}$. Além desses, Curitiba-PR ${ }^{(25)}$ e São Paulo-SP(24) estão presentes nos estudos, e apenas um não informou o local de sua coleta de $\operatorname{dados}^{(18)}$.

Com relação às populações estudadas, dois artigos apresentaram amostras representativas ${ }^{(23,25)}$ descrevendo os procedimentos para os respectivos cálculos. Dois estudos utilizaram uma coorte com nascidos em $1993^{(14,26)}$ e um avaliou todos os escolares da faixa etária de interesse ${ }^{(1)}$, porém, este obteve um baixo poder para determinadas análises. Por fim, em outros dois estudos as amostras utilizadas eram intencionais ${ }^{(18,24)}$.
Sobre os instrumentos utilizados para avaliar a AF, predominaram as avaliações autorreportadas, sendo estas utilizadas por quatro $\operatorname{artigos}^{(1,18,24,25)}$, dos quais um se utilizou do questionário "Estilo de vida fantástico"(18) e um optou pela versão longa do International physical activity questionnaire (IPAQ) $)^{(1)}$ para avaliar o NAF dos pais. Outros três estudos abordaram a entrevista estruturada como instrumento de medida da $\mathrm{AF}^{(14,23,26)}$. Não há um consenso sobre o tipo de AF analisada pelos artigos selecionados na atual revisão sistemática. Os instrumentos utilizados avaliaram a AF de suas amostras por diferentes maneiras, como demonstrado no Quadro 1.

Os estudos que utilizaram as atividades comuns à faixa etária como medida do NAF encontraram prevalências de baixa AF em percentis de 52,3 e $72,8^{(14)}, 56,5$ e $82,1^{(23)}$ e 37,4 e $65,5^{(26)}$, para sexo masculino e feminino, respectivamente, sendo que os dois últimos estudos também utilizaram a medida de deslocamento ativo para chegarem a esses resultados. Entre os pais, três estudos selecionados na presente revisão categorizaram a $\mathrm{AF}$ em ativo ou inativo, sendo seus resultados de inatividade em percentis 76,9 e $78,5^{(23)}$, para pai e mãe, respectivamente. Outros dois estudos não estratificaram os pais por sexo e encontraram

Quadro 1 - Metodologias empregadas na revisão sistemática da associação da atividade física de adolescentes, familiares e pares. Curitiba-PR, 2013-2014.

\begin{tabular}{|c|c|c|c|c|}
\hline Domínio da AF & Criança / Adolescente & Pais & Irmãos & Pares \\
\hline $\begin{array}{l}\text { Atividades comuns } \\
\text { à faixa etária }\end{array}$ & $\begin{array}{l}\text { Dumith et al. }(2012)^{(14)} \text {; Bastos, } \\
\text { Araújo, Hallal }(2008)^{(23)} \text {; Dumith et } \\
\text { al. }(2010)^{(26)}\end{array}$ & - & - & - \\
\hline Deslocamento ativo & $\begin{array}{l}\text { Bastos, Araújo, Hallal }(2008)^{(23)} \text {; } \\
\text { Duncan et al. }(2011)^{(24)} \text {; Dumith et al. } \\
(2010)^{(26)}\end{array}$ & - & - & - \\
\hline $\begin{array}{l}\text { Prática de atividades } \\
\text { esportivas }\end{array}$ & Duncan et al. $(2011)^{(24)}$ & - & - & - \\
\hline AFMV & Fermino et al. $(2010)^{(25)}$ & - & - & - \\
\hline Ativo / Inativo & Raphaelli, Azevedo, Hallal (2011) ${ }^{(1)}$ & $\begin{array}{l}\text { Petroski, Pelegrini } \\
(2009)^{(18)}\end{array}$ & $\begin{array}{l}\text { Duncan et al. } \\
(2011)^{(24)}\end{array}$ & - \\
\hline Estilo de vida & - & $\begin{array}{l}\text { Raphaelli, Azevedo, } \\
\text { Hallal (2011) } \\
\text { Dumith et al. (2012) } \\
\text { (14); Bastos, Araújo, } \\
\text { Hallal }(2008)^{(23)} ; \\
\text { Dumith et al. (2010) } \\
\text { (26) }\end{array}$ & - & - \\
\hline
\end{tabular}

AFMV: Atividade Física Moderada a Vigorosa 
Quadro 2 - Estudos que investigaram associação entre atividade física (AF) de adolescentes, pais, irmãos e amigos e seus principais resultados. Curitiba-PR, 2013-2014.

\begin{tabular}{|c|c|c|c|c|c|}
\hline Referência & $\begin{array}{l}\text { Design do } \\
\text { estudo }\end{array}$ & $\begin{array}{l}\text { Medida de } \\
\text { AF }\end{array}$ & $\begin{array}{l}\text { Associação } \\
\text { com }\end{array}$ & $\begin{array}{l}\text { Tratamento } \\
\text { estatístico }\end{array}$ & $\begin{array}{l}\text { Associação da AF de adolescentes e pais, irmãos } \\
\text { e/ou pares }\end{array}$ \\
\hline $\begin{array}{l}\text { Bastos, Araújo } \\
\text { e Hallal, } \\
2008^{(23)}\end{array}$ & Transversal & $\begin{array}{l}\text { Entrevista } \\
\text { estruturada }\end{array}$ & Pai e mãe & $\begin{array}{l}\text { Regressão de } \\
\text { Poisson }\end{array}$ & $\begin{array}{l}\text { Meninas com pais e mães inativos apresentam } \\
\text { maior probabilidade de serem inativas }(1,21 \text { e } 1,14 \text {, } \\
\text { respectivamente). Sem associação entre os meninos. }\end{array}$ \\
\hline $\begin{array}{l}\text { Dumith et al., } \\
2012^{(14)}\end{array}$ & $\begin{array}{l}\text { Longitudinal } \\
\text { prospectivo }\end{array}$ & $\begin{array}{l}\text { Auto } \\
\text { reportada }\end{array}$ & Mãe & $\begin{array}{l}\text { Regressão } \\
\text { múltipla }\end{array}$ & Não houve associação significativa. \\
\hline $\begin{array}{l}\text { Duncan et al., } \\
2011^{(24)}\end{array}$ & Transversal & $\begin{array}{l}\text { Auto } \\
\text { reportada }\end{array}$ & $\begin{array}{l}\text { Pai, mãe e } \\
\text { irmãos }\end{array}$ & $\begin{array}{l}\text { Regressão } \\
\text { logística }\end{array}$ & $\begin{array}{l}\text { Crianças e adolescentes com um dos pais praticantes } \\
\text { de atividade física apresentaram } 1,25 \text { vezes mais } \\
\text { chances de ter sobrepeso/obesidade em comparação } \\
\text { aos que não tinham nenhum dos pais praticantes. } \\
\text { Aqueles que receberam encorajamento de um } \\
\text { ou ambos os pais apresentaram } 1,67 \text { e } 1,63 \text { mais } \\
\text { chances de apresentarem sobrepeso/obesidade, } \\
\text { respectivamente, em relação aos que não recebiam. } \\
\text { Sem associação em relação aos irmãos inativos. }\end{array}$ \\
\hline $\begin{array}{l}\text { Dumith et al., } \\
2010^{(26)}\end{array}$ & Transversal & $\begin{array}{l}\text { Auto } \\
\text { reportada }\end{array}$ & Pai e mãe & $\begin{array}{l}\text { Regressão de } \\
\text { Poisson }\end{array}$ & $\begin{array}{l}\text { Meninas com pais e/ou mães ativos apresentam } \\
\text { probabilidade de } 1,50 \text { vezes maior de serem ativas } \\
\text { comparadas com filhas de pais inativos. Sem } \\
\text { associação para os meninos. }\end{array}$ \\
\hline $\begin{array}{l}\text { Fermino et al., } \\
2010^{(25)}\end{array}$ & Transversal & $\begin{array}{l}\text { Auto } \\
\text { reportada }\end{array}$ & $\begin{array}{l}\text { Família e } \\
\text { amigos }\end{array}$ & $\begin{array}{l}\text { Regressão de } \\
\text { Poisson }\end{array}$ & $\begin{array}{l}\text { Adolescentes que recebem apoio social da família } \\
\text { apresentam uma probabilidade de } 1,32 \text { e } 1,14 \text { vezes } \\
\text { maiores de serem fisicamente ativos em relação } \\
\text { aos que não recebem, para as análises bivariadas } \\
\text { e multivariadas respectivamente. Adolescentes que } \\
\text { recebem apoio social dos amigos apresentam uma } \\
\text { probabilidade de } 1,97 \text { e } 1,52 \text { vezes maiores de } \\
\text { serem fisicamente ativos em relação aos que não } \\
\text { recebem, para as análises bivariadas e multivariadas } \\
\text { respectivamente. Adolescentes que recebem apoio } \\
\text { social da família apresentam uma probabilidade de } \\
1,49 \text { de vezes maior de cumprir as recomendações } \\
\text { para a AF ( } \geq 60 \text { min/dia) em relação aos que não } \\
\text { recebem. Adolescentes que recebem apoio social } \\
\text { dos amigos apresentam uma probabilidade de } 2,44 \\
\text { vezes maior de cumprir as recomendações para a } \\
\text { AF ( } \geq 60 \text { min/dia) em relação aos que não recebem } \\
\text { apoio social dos amigos. }\end{array}$ \\
\hline $\begin{array}{l}\text { Petroski, } \\
\text { Pelegrini, } \\
2009^{(18)}\end{array}$ & Transversal & $\begin{array}{l}\text { Auto } \\
\text { reportada } \\
\text { (Estilo } \\
\text { de vida } \\
\text { fantástico) }\end{array}$ & Pai e mãe & $\begin{array}{l}\text { Exato de } \\
\text { Fischer }\end{array}$ & $\begin{array}{l}\text { Filhos com baixo percentual de gordura apresentam } \\
\text { seus pais com um estilo de vida mais favorável à } \\
\text { saúde. }\end{array}$ \\
\hline $\begin{array}{l}\text { Raphaelli, } \\
\text { Azevedo, } \\
\text { Hallal, 2011(1) }\end{array}$ & Transversal & $\begin{array}{l}\text { Auto } \\
\text { reportada } \\
\text { (Bastos et } \\
\text { al, 2008 } \\
\text { IPAQ versão } \\
\text { longa) }\end{array}$ & Pai e mãe & $\begin{array}{l}\text { Teste de qui- } \\
\text { quadrado e } \\
\text { regressão de } \\
\text { Poisson }\end{array}$ & $\begin{array}{l}\text { Associação entre a atividade física do pai e do } \\
\text { adolescente na análise bruta. Na ajustada, a } \\
\text { probabilidade de o adolescente ser ativo ficou em } \\
1,40 \text {. } \\
\text { Ao estratificar a análise por sexo do escolar, o pai } \\
\text { ser ativo esteve associado à maior chance de os } \\
\text { adolescentes do sexo feminino realizarem atividade } \\
\text { física. }\end{array}$ \\
\hline
\end{tabular}


prevalências de inativos em $84,7 \%^{(26)}$ e $48,5 \%^{(1)}$, e um estudo no qual dois terços das mães dos adolescentes não estavam envolvidas em AF em seu tempo de lazer ${ }^{(14)}$.

Em dois artigos ${ }^{(23,26)}$ a AF das filhas foi positivamente associada à $\mathrm{AF}$ dos pais, apresentando valores para a razão de prevalência de $1,21(1,01-1,46), 1,14(1,02-1,28)^{(23)} \mathrm{e}$ $1,50(1,31-1,72)^{(26)}$, e um estudo encontrou uma associação positiva para o adolescente e seu pai, tendo uma razão de prevalência de $1,40(1,10-180)^{(1)}$.

\section{DISCUSSÃO}

O fato de se encontrar poucos estudos brasileiros indexados nas bases de dados internacionais deixa clara a necessidade de pesquisas sobre a AF de adolescentes e suas relações com familiares e pares, corroborando com autores que informaram a necessidade de estudos sobre aspectos sociais e a $\mathrm{AF}^{(22)}$.

Sabe-se que a AF abaixo dos níveis recomendados é um dos comportamentos de risco mais comuns entre os adolescentes, com taxas de prevalência acima de $50 \%^{(27)}$, semelhantes aos resultados apresentados pelos artigos desta revisão, porém, abaixo da média global, que é de $80,3 \%$ dos adolescentes abaixo das recomendações para a $\mathrm{AF}^{(11)}$. Sobre o NAF dos pais, os estudos da atual revisão apresentam valores muito acima da média global, que é de $31,1 \%$ de adultos fisicamente inativos ${ }^{(11)}$. Essas diferenças podem estar relacionadas aos diferentes tipos de instrumentos utilizados para estimar a AF, além, claro, das diferenças socioculturais e distinções regionais que influenciam, dentre outras coisas, na prática de $\mathrm{AF}$.

A associação positiva entre a $\mathrm{AF}$ de adolescentes do sexo feminino e seus pais foi encontrada tanto para pais inativos e filhas inativas ${ }^{(23)}$ quanto para pais ativos e filhas ativas $^{(26)}$, o que poderia ser explicado pelo fato de que a $\mathrm{AF}$ dos pais poderia ter um maior efeito no sexo feminino do que no masculino ${ }^{(28)}$. Corroborando com outros estudos ${ }^{(1,26)}$, os quais mostraram que adolescentes do sexo feminino com pais e/ou mães ativos são $50 \%$ mais propensos ao estilo de vida ativo do que filhas de pais inativos, e a chance de o adolescente ser ativo quando seu pai também é ficou em $40 \%^{(1,26)}$.

Ainda sobre as associações, uma prévia revisão mostrou haver uma correlação entre o NAF de pais e filhos, e que um alto NAF paternal e maternal está associado a um alto NAF de adolescentes ${ }^{(29)}$, o que deixa claro o modelo familiar ao associar a prática da $\mathrm{AF}$ de pais e filhos ${ }^{(1,29)}$.

Um estudo longitudinal ligou sua baseline a três fatores relacionados à AF: percepção das atitudes parentais, do formato do corpo e aptidão pelos adolescentes do sexo masculino e atitudes parentais sobre AF, além da AF das mães ${ }^{(30)}$. Todavia, a análise longitudinal abordada na presente revisão mostrou não haver associação significativa entre a $\mathrm{AF}$ de mães e seus filhos ${ }^{(14)}$, mostrando que os resultados podem indicar influência dos pais quando em estudos de design transversal; ou, como no caso do estudo abordado por esta revisão, na baseline, mas não nas mudanças nos padrões de AF com o passar dos anos ${ }^{(30)}$.

Sobre a composição corporal, os dois estudos que abordaram esse tema como desfecho apresentam resultados discordantes. Crianças e adolescentes com um dos pais envolvidos em práticas esportivas apresentaram 1,25 (1,021,53) mais chances de terem sobrepeso/obesidade em relação aos que não tinham nenhum dos pais envolvidos em esportes; e nesse mesmo estudo, adolescentes que receberam encorajamento, de um ou ambos os pais, para a AF têm, respectivamente, 1,67 (1,26-2,32) e 1,63 (1,27-2,08) mais chances de apresentarem sobrepeso/ obesidade $^{(24)}$. Entretanto, a literatura fornece evidências de que o comportamento de risco dos pais está associado ao comportamento de risco dos filhos ${ }^{(31)}$, e também que filhos de pais ativos apresentam maior probabilidade de serem $\operatorname{ativos}^{(1,23,26,32) \text {. }}$

Esta atual revisão encontrou um estudo que associou a $\mathrm{AF}$ ao apoio social recebido pelo adolescente ${ }^{(25)}$, cujos resultados indicaram que adolescentes que receberam apoio social da família apresentam uma probabilidade de $1,32(1,18-1,47)$ e $1,14(1,05-1,23)$ vezes maiores de serem fisicamente ativos em relação aos que não recebem o apoio social da família, para as análises bivariadas e multivariadas, respectivamente. Sobre cumprir as recomendações para a $\operatorname{AF}(\geq 60 \mathrm{~min} / \mathrm{dia})$, as análises de razão de prevalência mostraram que o apoio social da família aumenta as probabilidades em 1,49 $(1,10-2,02)^{(25)}$.

Esses resultados estão de acordo com as evidências encontradas na literatura, as quais sugerem que familiares representam um importante papel no desenvolvimento de comportamentos saudáveis por parte de seus filhos ${ }^{(33)}$, seja pelo envolvimento direto ou servindo como modelo para um comportamento ativo ${ }^{(19)}$. Vale ressaltar que os pais têm a possibilidade de condicionar o acesso a equipamentos e a envolvimentos que facilitem a $\mathrm{AF}$, diminuindo, assim, algumas barreiras, como não ter alguém para levar, por exemplo ${ }^{(34)}$. Aliado a isso, há uma associação positiva entre o comportamento dos pais e o de seus filhos, seja este sedentário ou fisicamente ativo ${ }^{(25)}$.

Também foi observado que receber apoio social dos amigos representa uma probabilidade de 1,97 (1,67$2,33)$ e $1,52(1,31-1,78)$ vezes maior para ser fisicamente ativo em relação a quem não o recebe, para as análises bivariadas e multivariadas, respectivamente. Com relação a cumprir as recomendações para a $\mathrm{AF}$, as probabilidades foram aumentadas em 2,44 $(1,67-3,57)$ com o apoio social dos amigos ${ }^{(25)}$. Isso pode ser explicado pela tendência de 
adolescentes ativos terem amigos igualmente $\operatorname{ativos}^{(32)}$, apresentando NAFs associados entre $\mathbf{s i}^{\prime(35)}$, pois sabe-se que adolescentes influenciam-se mutuamente no iniciar de uma atividade e na continuidade desta ${ }^{(36)}$, portanto, um maior contato com os amigos, em especial fora do ambiente escolar, parece contribuir para um comportamento ativo ${ }^{(25)}$. Complementando esse esclarecimento, além da associação com os indicadores de influência, existe uma associação positiva entre AF, o NAF dos amigos e a presença destes durante as atividades ${ }^{(21)}$. Assim, fica evidente a importância de amigos ativos, afinal, a adolescência é um período da vida caracterizado pelo aumento da independência da família e expansão das redes sociais externas ${ }^{(37)}$.

$\mathrm{O}$ apoio social surge, então, como um consistente e importante determinante para a AF, especialmente o suporte parental e dos amigos, por sua positiva associação com o NAF dos adolescentes ${ }^{(38)}$.

Dentre as limitações observadas nos sete estudos que fizeram parte da presente revisão sistemática, um dos estudos não estratificou sua amostra por $\operatorname{sexo}^{(23)}$, e outro fez uso de um questionário não validado ${ }^{(24)}$. $\mathrm{O}$ fato de as amostras serem de estudantes da rede pública de ensino na maioria dos estudos limita a compreensão dos fenômenos estudados em diferentes níveis socioculturais ${ }^{(25)}$; e o fato de apenas um dos pais aceitar participar do estudo ${ }^{(1)}$ e outro avaliar apenas as mães ${ }^{(14)}$, além de outro estudo utilizar uma amostra intencional ${ }^{(18)}$ também é limitador. Outra grande limitação comum a todos os estudos é que nenhum controlou a maturação sexual em suas análises. É importante destacar, também, a dificuldade de se comparar os estudos, por utilizarem diferentes instrumentos na mensuração da AF. Desse modo, existem lacunas a serem respondidas por futuras investigações, além da necessidade de novos estudos sobre a temática.

\section{CONCLUSÃO}

Com base nos achados da presente revisão, verificou-se que existe uma associação positiva entre a atividade física do pai e da mãe com suas filhas, tanto para a inatividade quanto para serem ativos. Porém, não foram encontradas associações entre ambos os pais e os filhos, fato também observado para com irmãos inativos. Quanto à composição corporal, os resultados são contraditórios, com um estudo apontando para filhos de pais envolvidos em atividades esportivas com maior probabilidade de apresentarem sobrepeso e/ou obesidade, e outro estudo no qual pais com estilo de vida mais favorável à saúde têm seus filhos com baixos percentis de gordura. Além disso, o apoio social mostrou-se eficaz para aumentar o nível de atividade física de adolescentes.

\section{REFERÊNCIAS}

1. Raphaelli CO, Azevedo MR, Hallal PC. Association between health risk behaviors in parents and adolescents in a rural area in southern Brazil. Cad Saúde Pública. 2011;27(12):2429-40.

2. Babey SH, Wolstein J, Krumholz S, Robertson B, Diamant AL. Physical activity, park access and park use among California adolescents. UCLA Centre for Health Policy Research. 2013 Mar(PB2013-2):1-8.

3. Logstein B, Blekesaune A, Almas R. Physical activity among Norwegian adolescents--a multilevel analysis of how place of residence is associated with health behaviour: the Young-HUNT study. Int J Equity Health. 2013;12:56.

4. Carson V, Rosu A, Janssen I. A cross-sectional study of the environment, physical activity, and screen time among young children and their parents. BMC Public Health. 2014;14(1):61.

5. Young DR, Reynolds K, Sidell M, Brar S, Ghai NR, Sternfeld B, et al. Effects of physical activity and sedentary time on the risk of heart failure. Circ Heart Fail. 2014;7(1):21-7.

6. Swift DL, Johannsen NM, Lavie CJ, Earnest CP, Church TS. The role of exercise and physical activity in weight loss and maintenance. Prog Cardiovasc Dis. 2014;56(4):441-7.

7. Rasberry CN, Lee SM, Robin L, Laris BA, Russell LA, Coyle KK, et al. The association between schoolbased physical activity, including physical education, and academic performance: a systematic review of the literature. Prev Med. 2011;52 Suppl 1:S10-20.

8. Lima DF, Levy RB, Luiz OC. Recomendações para atividade física e saúde: consensos, controvérsias e ambiguidades. Rev Panam Salud Publica. 2014;36(3):164-70.

9. Haskell WL, Lee IM, Pate RR, Powell KE, Blair SN, Franklin BA, et al. Physical activity and public health: updated recommendation for adults from the American College of Sport Medicine and the American Heart Association. Med Sci Sport Exerc. 2007;3(2):1423-34.

10. International Physical Activity Questionnaire (IPAQ). Guidelines for data processing and analysis of the International Physical Activity Questionnaire (IPAQ) short and long forms. 2005. [acesso em 2015 Jan 21]. Disponível em: https://www.academia.edu/5346814/ Guidelines_for_Data_Processing_and_Analysis_of the_International_Physical_Activity_Questionnaire IPAQ_Short_and_Long_Forms_Contents 
11. Hallal PC, Andersen LB, Bull FC, Guthold R, Haskell W, Ekelund U. Global physical activity levels: surveillance progress, pitfalls, and prospects. Lancet. 2012;21;380(9838):247-57.

12. Knowles AM, Niven A, Fawkner S. A qualitative examination of factors related to the decrease in physical activity behavior in adolescent girls during the transition from primary to secondary school. J Phys Act Health. 2011;8(8):1084-91.

13. Corder K, Atkin AJ, Ekelund U, van Sluijs EM. What do adolescents want in order to become more active? BMC Public Health. 2013;13(1):718.

14. Dumith SC, Gigante DP, Domingues MR, Hallal PC, Menezes AM, Kohl HW, 3rd. Predictors of physical activity change during adolescence: a 3.5 -year followup. Public Health Nutr. 2012;15(12):2237-45.

15. Guedes DP, Souza MV, Ferreirinha JE, SilvaAJ. Physical activity and determinants of sedentary behavior in Brazilian adolescents from an underdeveloped region. Percept Mot Skills. 2012;114(2):542-52.

16. Guimarães RF, Silva MP, Legnani E, Mazzardo O, Campos W. Reproducibility of adolescent sedentary activity questionnaire (ASAQ) in Brazilian adolescents. Rev Bras Cineantropom Desempenho Hum. 2013;15(3):276-85.

17. Maher C, Olds T, Mire E, Katzmarzyk PT. Reconsidering the sedentary behaviour paradigm. PLoS One. 2014;9(1):e86403.

18. Petroski EL, Pelegrini A. Associação entre o estilo de vida dos pais e a composição corporal dos filhos adolescentes. Rev Paul Pediatr. 2009;27(1):48-52.

19. O’Connor TM, Jago R, Baranowski T. Engaging Parents to Increase Youth Physical Activity: A Systematic Review. Am J Prev Med. 2009;37(2):141-9.

20. Horimoto AR, Giolo SR, Oliveira CM, Alvim RO, Soler JP, de Andrade M, et al. Heritability of physical activity traits in Brazilian families: the Baependi Heart Study. BMC Med Genet. 2011;12:155.

21. Maturo CC, Cunningham SA. Influence of friends on children's physical activity: a review. Am J Public Health. 2013;103(7):e23-38.

22. Bauman AE, Reis RS, Sallis JF, Wells JC, Loos RJ, Martin BW. Correlates of physical activity: why are some people physically active and others not? Lancet. 2012;380(9838):258-71.

23. Bastos JP, Araujo CL, Hallal PC. Prevalence of insufficient physical activity and associated factors in Brazilian adolescents. J Phys Act Health. 2008;5(6):777-94.

24. Duncan S, Duncan EK, Fernandes RA, Buonani C, Bastos KD, Segatto AF, et al. Modifiable risk factors for overweight and obesity in children and adolescents from Sao Paulo, Brazil. BMC Public Health. 2011;11:585.

25. Fermino RC, Rech CR, Hino AA, Rodriguez Anez CR, Reis RS. Physical activity and associated factors in high-school adolescents in Southern Brazil. Rev Saúde Pública. 2010;44(6):986-95.

26. Prevalence and correlates of physical activity among adolescents from Southern Brazil. Rev Saúde Pública. 2010;44(3):457-67.

27. Barbosa Filho VC, Campos W, Bozza R, Lopes AS. The prevalence and correlates of behavioral risk factors for cardiovascular health among Southern Brazil adolescents: a cross-sectional study. BMC Pediatr. 2012;12:130.

28. O'Loughlin J, Paradis G, Kishchuk N, Barnett T, Renaud L. Prevalence and correlates of physical activity behaviors among elementary schoolchildren in multiethnic, low income, inner-city neighborhoods in Montreal, Canada. Ann Epidemiol. 1999;9(7):397-407.

29. Gustafson SL, Rhodes RE. Parental correlates of physical activity in children and early adolescents. Sports Med. 2006;36(1):79-97.

30. Kahn JA, Huang B, Gillman MW, Field AE, Austin SB, Colditz GA, et al. Patterns and Determinants of Physical Activity in U.S. Adolescents. J Adolesc Health. 2008;42(4):369-77.

31. Wickrama KA, Conger RD, Wallace LE, Elder GH, Jr. The intergenerational transmission of health-risk behaviors: adolescent lifestyles and gender moderating effects. Journal of health and social behavior. 1999 Sep;40(3):258-72.

32. Seabra AF, Mendonca DM, Thomis MA, Anjos LA, Maia JA. Biological and socio-cultural determinants of physical activity in adolescents. Cad Saúde Pública. 2008;24(4):721-36.

33. Trost SG, Sallis JF, Pate RR, Freedson PS, Taylor WC, Dowda M. Evaluating a model of parental influence on youth physical activity. Am J Prev Med. 2003;25(4):277-82.

34. Santos MS, Hino AA, Reis RS, Rodriguez-Anez CR. Prevalence of barriers for physical activity in adolescents. Rev Bras Epidemiol. 2010;13(1):94-104. 
35. Force CPST. Improving adolescent health through interventions targeted to parents and other caregivers: a recommendation. Am J Prev Med. 2012;42(3):327-8.

36. Pratt M, Sarmiento OL, Montes F, Ogilvie D, Marcus BH, Perez LG, et al. The implications of megatrends in information and communication technology and transportation for changes in global physical activity. Lancet. 2012;380(9838):282-93.

37. Parra DC, Hoehner CM, Hallal PC, Ribeiro IC, Reis R, Brownson RC, et al. Perceived environmental correlates of physical activity for leisure and transportation in Curitiba, Brazil. Prev Med. 2011;52(3-4):234-8.
38. Park H, Kim N. Predicting Factors of Physical Activity in Adolescents: A Systematic Review. Asian Nurs Res (Korean Soc Nurs Sci). 2008;2(2):113-28.

\section{Endereço para correspondência:}

Thiago Silva Piola

Universidade Federal do Paraná

Rua Coração de Maria, 92

Bairro: Jardim Botânico

CEP: 80215-370 - Curitiba - PR - Brasil

E-mail: tspthiago@hotmail.com 